\title{
Closed-Loop Global Motion Planning for Reactive, Collision-Free Execution of Learned Tasks
}

\author{
CHRIS BOWEN and RON ALTEROVITZ, University of North Carolina at Chapel Hill, USA
}

\begin{abstract}
We present a robot motion planning approach for performing a learned task while reacting to the movement of obstacles and task-relevant objects. We employ a closed-loop, sampling-based motion planner operating multiple times a second that senses obstacles and task-relevant objects and generates collision-free motion plans based on a learned-task model. The task model is learned from expert demonstrations prior to task execution and is represented as a hidden Markov model. During task execution, our motion planner quickly searches in the Cartesian product of the task model and a probabilistic roadmap for a collision-free plan with features most similar to the demonstrations given the current locations of the task-relevant objects. We accelerate replanning using a fast bidirectional search and by biasing the sampling distribution using information from the learned-task model. We show the efficacy of our approach with the Baxter robot performing two tasks.
\end{abstract}

CCS Concepts: • Computer systems organization $\rightarrow$ Robotic autonomy; External interfaces for robotics;

Additional Key Words and Phrases: Interactive motion planning, asymptotically optimal motion planning, assistive robotics

\section{ACM Reference format:}

Chris Bowen and Ron Alterovitz. 2018. Closed-Loop Global Motion Planning for Reactive, Collision-Free Execution of Learned Tasks. ACM Trans. Hum.-Robot Interact. 7, 1, Article 10 (May 2018), 16 pages.

https://doi.org/10.1145/3209045

\section{INTRODUCTION}

Robotic manipulators have the potential to assist people with a variety of routine tasks in homes and workplaces. One key challenge for autonomously completing tasks in environments where people live and work is that objects in the environment are often not static-objects relevant to a task may move independently or be moved by people. A second key challenge is that many tasks must be performed in a manner that enforces constraints that humans are aware of from context and intuition: for example, the constraint that a glass of water must be kept approximately level when being carried in order to avoid spilling the water. A third key challenge is that homes and workspaces are often cluttered with (possibly moving) obstacles that must be avoided to safely perform a task.

This research was supported in part by the National Science Foundation (NSF) under award nos. IIS-1149965 NSF and CCF-1533844.

Authors' address: C. Bowen and R. Alterovitz, Department of Computer Science, University of North Carolina at Chapel Hill, 201 S. Columbia St., Chapel Hill, NC, 27599-3175, USA; emails: \{cbbowen, ron\}@cs.unc.edu.

Permission to make digital or hard copies of part or all of this work for personal or classroom use is granted without fee provided that copies are not made or distributed for profit or commercial advantage and that copies bear this notice and the full citation on the first page. Copyrights for third-party components of this work must be honored. For all other uses, contact the owner/author(s).

(c) 2018 ACM 2573-9522/2018/05-ART10 $\$ 15.00$

https://doi.org/10.1145/3209045 

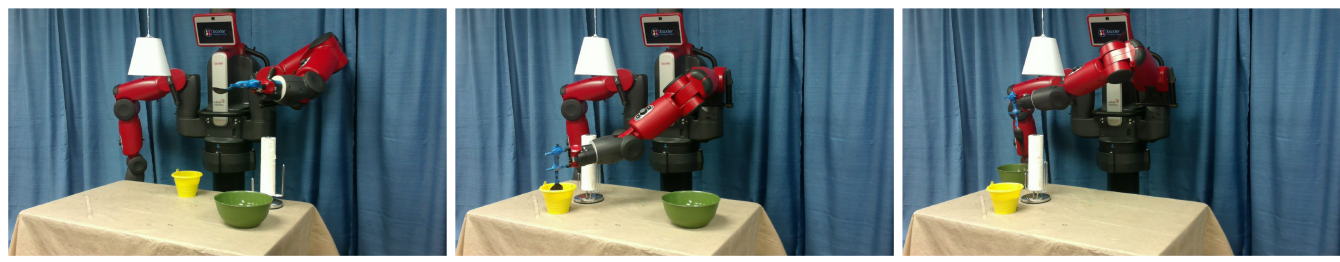

Fig. 1. As the Baxter robot performs the learned task of transferring powder from the yellow bucket to the green bowl using the blue spoon, a person moves the green bowl. Our method automatically replans in a closed-loop manner, enabling the robot to avoid obstacles and perform the learned task even when taskrelevant objects or obstacles are moved mid-task.

In this article, we present a motion planning approach that addresses each of these challenges by using a learned task model and executing in a closed-loop manner, enabling the robot to be responsive to the motion of task-relevant objects and obstacles that should be avoided.

To learn a task model, our method requires a set of human-guided demonstrations, which can be performed in a static environment with no obstacles present. If the task-relevant objects are in different locations in different demonstrations, then the learner can generalize the task model to new locations. Once the task is learned, our motion planner can be executed in new environments with novel obstacles and can adjust to the movement of these obstacles and task-relevant objects while the task is being performed.

Based on prior work $[7,44]$, we consider a task model consisting of a sequence of probability distributions over the configuration of the robot given the state of the environment. Specifically, we consider features defined by both the configuration of the robot and the position of the end-effector relative to task-relevant objects (e.g., the green bowl in the powder transfer task in Figure 1). We learn not only the average values of these features over time but also their variances, under the assumption that more consistent features in the demonstrations should similarly be reproduced consistently during execution. For instance, in the powder-transfer task in Figure 1, the human demonstrator likely kept the orientation of the spoon consistently level while it contained powder, and consequently the robot should reproduce this feature.

During execution, our motion planner uses these learned distributions to define a cost function that is minimized by an interactive-rate motion planner. We leverage a sampling-based motion planner that uses these learned distributions to guide sampling toward low-cost regions by incorporating the positions of task-relevant obstacles. The solutions found by the motion planner approach global optimality in contrast to approaches that guarantee only local optimality and thus may become caught in the basin of attraction of a bad solution. We search for plans in a roadmap defined by the Cartesian product of the learned-task model, represented as a hidden Markov model, and a probabilistic roadmap over the robot's configuration space.

To consider the movement of task-relevant objects, we continuously replan by rebuilding the roadmap and then searching for a new plan. This replanning approach computes plans that avoid obstacles while explicitly considering task-relevant objects based on the current state of the environment. The robot replans in real time, averaging more than 5 plans per second in our experiments, by leveraging information in the task model and using appropriate data structures and algorithms. First, we employ a sampling distribution biased toward low-cost regions of configuration space to produce a small, high-quality roadmap. Second, we use a parallel bidirectional search over an implicit graph combined with lazy computation to quickly and globally search for optimal plans. 
In this article, in addition to providing a refined archival version of our results in [6], we present several important extensions. First, we further accelerated the motion planning by combining information from the environment and task model during sampling to enable the robot to react not only to the motion of task-relevant objects (as in [6]) but also to moving obstacles that are sensed using Kinect in real time. Second, we conducted experiments in more challenging scenarios with the Baxter robot in which multiple task-relevant objects and obstacles moved during execution and in which these obstacles could not easily be modeled using geometric primitives (e.g., plants), highlighting the need for closed-loop motion planning using sensed depth maps directly (e.g., from an RGBD sensor). Our results show that closed-loop global motion planning, enabled by unifying learning from demonstrations with fast sampling-based motion planning, can help enable a robot to autonomously perform learned tasks in environments with moving objects and obstacles.

\section{RELATED WORK}

Sampling-based methods have been highly successful for computing feasible (and optimal) motion plans for a wide variety of robots, including manipulators with many degrees of freedom $[10,26,32]$. While most sampling-based motion planners that consider optimality aim to minimize metrics such as Euclidean distance in the workspace or configuration space, some methods have investigated incorporating more general task-based cost functions. Several approaches are based on rapidly exploring random trees (RRTs) [32], a highly successful sampling-based method for computing feasible, obstacle-avoiding trajectories. Transition-based RRT (T-RRT) [23] biases expansion of an RRT to low-cost regions of the configuration space cost map. Mainprice et al. used T-RRT to generate natural motions based on a predefined cost map for human robot interaction [34]. RRTs have also been used in conjunction with analytically defined task constraints [42] and with symbolic representations of manipulation strategies [25]. Sampling-based motion planners have also investigated integrating motion constraints and properties learned from demonstrations. Claasens extended RRT to sample only inside a user-specified number of standard deviations of a mean demonstrated trajectory [11] and later within learned affordances [12], Berenson et al. integrated local optimization with an RRT to find low-cost paths over cost maps [3], Scholz and Stilman incorporated gradient descent into an RRT to locally optimize a specified objective function [39], and Şucan and Chitta investigated sampling strategies that enforce constraints [13]. Finally, RRTs have been used for replanning when the environment changes [19, 21, 45]. However, plans produced by RRT methods are almost surely suboptimal even as computation time approaches infinity. This limitation also applies to some roadmap methods [27], such as elastic roadmaps [43], which have been applied to the problem of motion planning in the presence of soft constraints.

We employ an asymptotically optimal sampling-based motion planner, meaning that the computed plan is guaranteed to approach a globally optimal plan (based on the given cost metric) as the number of iterations is allowed to increase. Karaman and Frazzoli proposed asymptotically optimal motion planning algorithms such as $\mathrm{RRG}^{*}$ and $\mathrm{PRM}^{*}$ that guarantee asymptotic optimality [26]. Asymptotically optimal motion planners avoid the suboptimal plans resulting from local minima that can occur when using potential field methods [10] or local optimizers [46] or resulting from sampling-based planners not designed for asymptotic optimality, such as RRT [26]. Related work has investigated asymptotically optimal planners that balance exploration and refinement [1], asymptotic near optimal planners using smaller roadmaps [35], the near-optimality of solutions in finite time [17], and anytime solution optimization [33].

Our method integrates a learned hidden Markov model (HMM) representing a task with a sampling-based motion planner to guarantee asymptotic optimality. HMMs have previously been used for motion generation $[9,30]$, but prior approaches, unlike our proposed method, do not simultaneously guarantee global optimality while enabling fast replanning. Motion planners that 
operate entirely on constraint manifolds have also been developed [4, 29] even while retaining asymptotic optimality $[8,24]$. However, statistical task models learned from demonstrations like the HMM we consider naturally encode uncertainty, and formulating hard constraints discards this information. Model predictive control approaches have also considered time-varying cost functions and may use sampling to avoid obstacles [20]. A number of methods learn high-level tasks from demonstrations, for example, $[2,36]$. For execution, these methods may use visual servoing in conjunction with subtask-specific motion planners [18] or more general constraints [31]. Rather than competing with these methods, our approach is more appropriate for learning and executing the subtasks on which such methods rely. These subtasks could then be combined to solve more complex tasks using general frameworks based on generic task planners [41], SMT solvers [14], or geometric backtracking [5]. Dynamical systems have also been used to learn such motions from demonstrations, for example, [22], [37], and [40]. These systems have further been extended to avoid obstacles in [28], but this approach retains no notion of global optimality.

\section{METHOD OVERVIEW}

\subsection{Problem Statement}

Let $Q \subset \mathbb{R}^{d}$ be the $d$-dimensional configuration space of a holonomic robot. Let $Q_{\text {free }} \subseteq Q$ denote the subset of the configuration space for which the robot is not in collision with an obstacle in the current execution environment. We assume that the robot is capable of sensing the positions of $K$ task-relevant objects, called landmarks (such as the green bowl in Figure 1), and a model of the environment against which we can test for collisions with obstacles. In our physical experiments, both landmark and obstacle sensing rely on a Kinect RGBD sensor.

During execution (described in Section 4), our objective is to compute a trajectory $\Phi$ in the robot's configuration space from a start configuration $\mathrm{q}_{\text {start }} \in Q_{\text {free }}$ to a goal configuration $\mathrm{q}_{\mathrm{goal}} \in$ $Q_{\text {free }}$ such that the trajectory (1) avoids obstacles in the robot's current environment and (2) successfully accomplishes the task, which may depend on task-relevant objects in the environment.

To address this challenge, our approach consists of two major phases: learning a task model from a set of demonstrations followed by task performance in the execution environment. Note that the task model is not tied to a specific environment; thus, it needs to be learned only once per task. It can then be used for execution across many environments even if task-relevant objects are in different places and new obstacles are present. Figure 2 illustrates an overview of the approach.

During the learning phase, the user provides demonstrations of a task class. These demonstrations can be performed with no obstacles present, and the environment in each demonstration can be static. As introduced in [44] and summarized in Section 3.2, we use statistical methods to learn a task model, which can be mapped to new environments with previously unseen obstacles and in which task-relevant objects may be in different locations.

In the execution phase, presented in Section 4, the robot continually senses its environment to collect sufficient information to perform collision detection and to compute costs in the current environment based on the learned-task model. The robot uses this information to compute collision-free motion plans based on the learned costs. We then enter the loop shown in red in Figure 2; the robot executes the current plan while, in a closed-loop manner, replanning based on the learned costs as task-relevant objects and obstacles might move in the environment.

\subsection{Learned-Task Model}

To learn a task, we consider a reformulation of the method originally presented in [7], wherein a task was learned from a set of demonstrations of a human kinesthetically guiding the robot through the task. For each demonstration, we record a sequence of configurations evenly spaced in time 


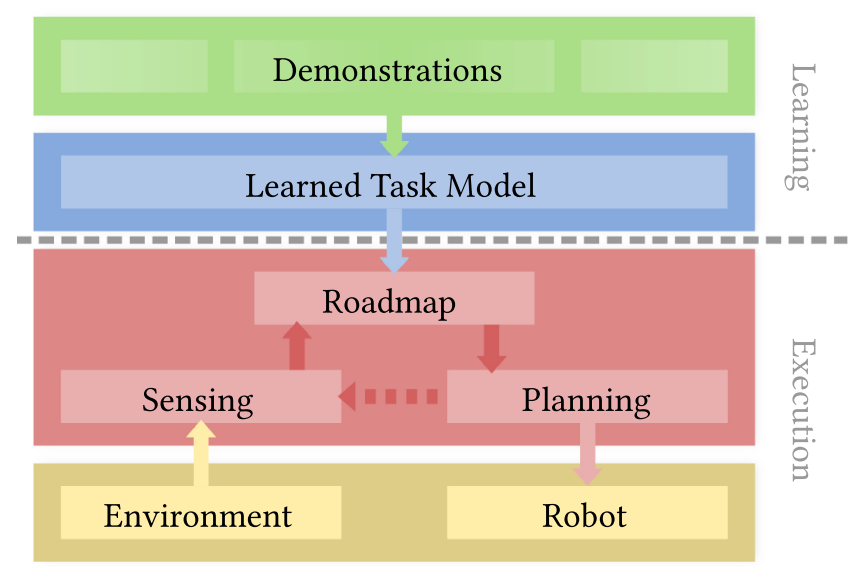

Fig. 2. General overview of the components of the system, with human input shown in green, task learning shown in blue, execution shown in red, and environment shown in yellow. Note that the task model is not specific to an environment and thus needs to be learned only once per task. It can then be used for execution across many environments. All arrows indicate execution flow and solid arrows indicate dataflow.

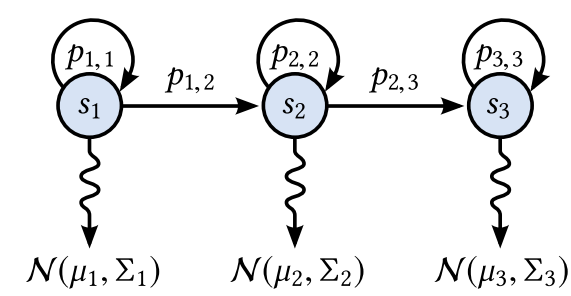

Fig. 3. Hidden Markov model with 3 discrete states (corresponding to timesteps of the task) and normally distributed observations.

and an annotation a that encodes the poses of task-relevant objects during the demonstration. The recorded robot configurations are then lifted via a function $f_{\mathrm{a}}(\mathbf{q})$ from configuration space into a motion feature space $\mathcal{Y} \subseteq \mathbb{R}^{m}$ comprising the robot's end-effector pose relative to task-relevant objects in the environment. We then time align the demonstrations and use them to estimate a sequence of multivariate normal distributions $\mathcal{N}\left(\mu_{t}, \Sigma_{t}\right)$ in the motion feature space.

In this article, for ease of integration with a constantly updating motion planning roadmap, we reformulate this approach as learning a time-homogeneous HMM with a restricted structure (see Figure 3). We assume that the model has $S$ discrete, sequential states wherein each state $s_{i}$ has nonzero transition probabilities only to itself and the next state $s_{i+1}$. This imparts a total ordering on the model corresponding to time. As such, we refer to these states as timesteps. For our experiments, we let the observed outputs in each state $s_{i}$ be distributed according to a multivariate Gaussian distribution $\mathcal{N}\left(\mu_{t}, \Sigma_{t}\right)$ in motion feature space. We learned the parameters of this model using the Viterbi path-counting algorithm [15].

This learned model will be used to compute a cost function for motion planning during task execution. The cost function is parameterized by the annotation a, which will vary across execution environments and during execution when a task-relevant object moves. The cost function [44] is defined such that a trajectory that minimizes it maximizes the likelihood in the learned model, and thereby should successfully perform the task in the execution environment with high probability. 


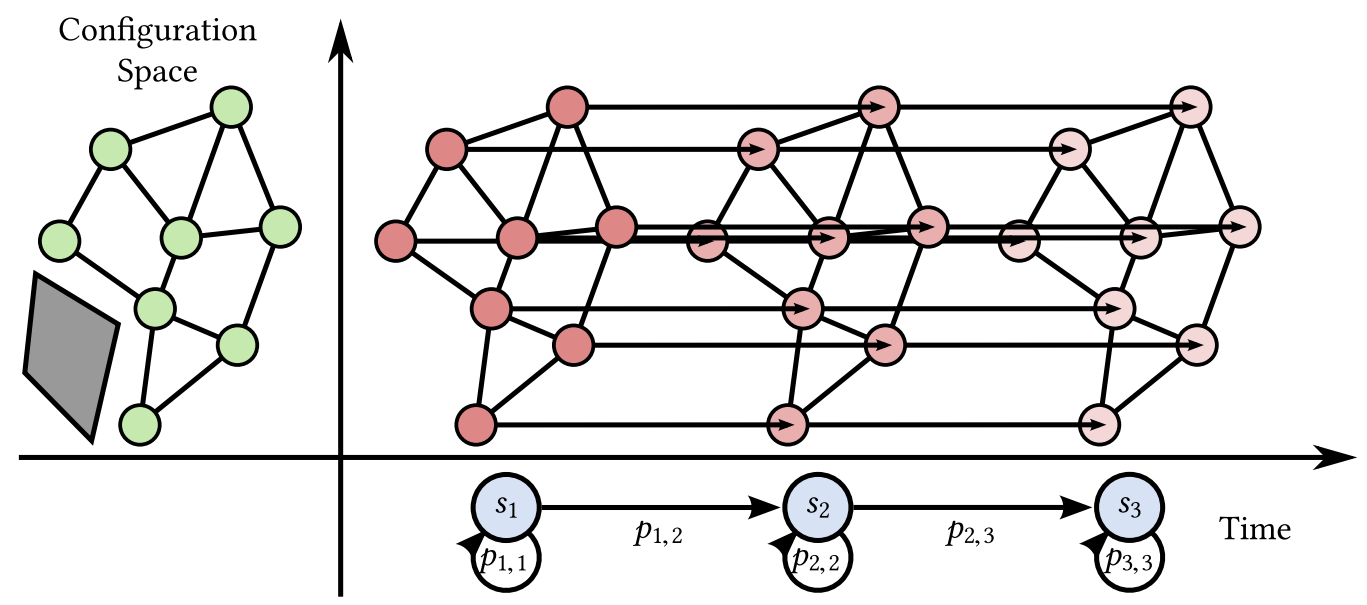

Fig. 4. Cartesian product of the configuration space roadmap and the graph of the learned-task model.

\section{CLOSED-LOOP REPLANNING WITH LEARNED COSTS}

In each replanning step, our method builds a spatiotemporal roadmap in which the edge costs are set based on the learned-task model and execution environment. We perform a shortest-path search in this roadmap to update the execution paths. We now describe each of the components of the closed-loop motion planning approach.

\subsection{Spatiotemporal Roadmap}

We employ an asymptotically optimal variation of a probabilistic roadmap with a fixed connection radius (sPRM) [27], which we chose to adapt because it produces globally optimal plans to within the roadmap resolution. A roadmap is a graph in which vertices represent the states of the robot and edges represent feasible local plans between these states. In the simplest case, these local plans are straight-line trajectories in configuration space.

A traditional roadmap is undirected and is constructed as follows. First, $n$ configurations $\left\{\mathbf{q}_{0}, \ldots, \mathbf{q}_{n}\right\}$ are randomly sampled from $Q_{\text {free }}$ via rejection sampling. Then, edges are constructed between all configurations $\mathbf{q}_{i}$ and $\mathbf{q}_{j}$ for which $\left\|\mathbf{q}_{i}-\mathbf{q}_{j}\right\|_{D}<\epsilon$ if a feasible local plan can be found. We construct exactly such a roadmap, which we will call the spatial roadmap.

To accommodate dependence on the timestep in the learned task, we also define a temporal roadmap. This is not a roadmap in the traditional sense but rather the graph representation of the Markov chain implied by the task model, in which timesteps correspond to vertices in the graph. Because the transitions in the Markov chain are directed, the temporal roadmap is a directed graph.

Finally, we define a spatiotemporal roadmap, a directed graph that combines the information in the spatial and temporal roadmaps. The vertices of the spatiotemporal roadmap are each defined by a pair composed of a vertex from the spatial roadmap and a vertex from the temporal roadmap. The set of edges are given by the vertex-wise union of edges in the spatial and temporal roadmaps (see Figure 4). Put another way, the spatiotemporal roadmap is the Cartesian product of the spatial and temporal roadmaps. Such a roadmap is necessary because the state of the robot needs to incorporate the task progress (see Section 5.1).

This full roadmap can be quite large, but because of its regular structure, it need not be explicitly constructed. Rather, we can implicitly traverse it by keeping track of vertices in the constituent roadmaps. This approach has multiple advantages over directly sampling in the product of 
configuration space and time. First, because the full roadmap does not need to be explicitly constructed, it uses less memory and exhibits better locality of reference. Second, because every vertex and edge in the spatial roadmap is effectively duplicated across all timesteps, fewer collision queries are required. Finally, small additions to either constituent roadmap are immediately reflected as larger additions to the full roadmap without the need to perform an explicit construction, making updates fast. As an aside, we also experimented with the tensor product of the roadmaps, which, while more natural, had many more edges and adversely impacted search performance.

\subsection{Cost Function}

In a traditional roadmap, the goal is to find shortest paths; thus, edges in the roadmap are assigned costs based on the lengths of the local plans that they represent. We wish to find paths that are most likely to successfully perform the task; thus, we choose edge costs based on the learned-task model. Specifically, we define a notion of cost that, when minimized, maximizes probability of successfully executing the task as defined by the learned model.

By the construction of the Cartesian product, each edge is from a configuration $\mathbf{q}$ and timestep $s$ to another configuration $\mathrm{q}^{\prime}$ and timestep $s^{\prime}$. Our definition of edge cost will be based on the negative $\log$ probability of entering timestep $s^{\prime}$ and observing configuration $\mathbf{q}^{\prime}$ after being in timestep $s$ and observing configuration $q$. The Markov assumption inherent in the task model implies that the configuration and subsequent timestep depend on the current timestep. This allows the following simplification in the edge cost computation:

$$
\begin{aligned}
-\log \left(p\left(\mathbf{q}^{\prime}, s^{\prime} \mid \mathbf{q}, s\right)\right) & = \\
-\log \left(p\left(\mathbf{q}^{\prime} \mid s^{\prime}\right) \cdot \mathrm{P}\left(s^{\prime} \mid s\right)\right) & =\quad \text { (Markov Property) } \\
-\log p\left(\mathbf{q}^{\prime} \mid s^{\prime}\right)-\log \mathrm{P}\left(s^{\prime} \mid s\right) &
\end{aligned}
$$

where the probability $\mathrm{P}\left(s^{\prime} \mid s\right)$ is given by $p_{s, s^{\prime}}$ and the probability density $p\left(\mathrm{q}^{\prime} \mid s^{\prime}\right)$ by

$$
\left|2 \pi \Sigma_{s^{\prime}}\right|^{-\frac{1}{2}} e^{-\frac{1}{2}\left(f_{\mathrm{a}}\left(\mathrm{q}^{\prime}\right)-\mu_{s^{\prime}}\right)^{\mathrm{T}} \Sigma_{s^{\prime}}^{-1}\left(f_{\mathrm{a}}\left(\mathrm{q}^{\prime}\right)-\mu_{s^{\prime}}\right)} .
$$

We consider the log probability because the shortest path algorithm will minimize the sum of the edge costs while joint probabilities (under independence assumptions) are multiplicative, as seen above. We then negate the result to formulate the problem as one of minimization. However, recall that edges in the spatial roadmap represent local plans. Thus, for such edges, we use the line integral of the negative log probability along the local plan $\mathbf{q}(t)$, yielding:

$$
\begin{gathered}
\operatorname{cost}\left(\mathbf{q}(\cdot), s, s^{\prime}\right)=\log \mathrm{P}\left(s^{\prime} \mid s\right)-\int_{0}^{1} \log p\left(\mathbf{q}(t) \mid s^{\prime}\right) \mathrm{d} t \\
=\log p_{s, s^{\prime}}-\frac{1}{2} \int_{0}^{1} \log m \log (2 \pi)+\log \left|\Sigma_{s^{\prime}}\right|+\left(f_{\mathbf{a}}\left(\mathbf{q}^{\prime}\right)-\mu_{s^{\prime}}\right)^{\mathrm{T}} \Sigma_{s^{\prime}}^{-1}\left(f_{\mathbf{a}}\left(\mathbf{q}^{\prime}\right)-\mu_{s^{\prime}}\right) \mathrm{d} t,
\end{gathered}
$$

where the local plan is parameterized in the interval $t \in[0,1]$.

When this cost is strictly positive, which can be ensured by appropriate choice of model like the one we use, asymptotic optimality is guaranteed by the sPRM method because a fixed connection radius using any $L^{p}$-norm is a superset of some other fixed radius using this cost metric.

\subsection{Biased Sampling}

Although asymptotic optimality ensures that the method converges to the optimal solution, this convergence may be too slow to achieve reactive execution. To accelerate this convergence, when sampling configurations to add to the roadmap, we use a biased sampling distribution rather than a uniform one. We construct the distribution in such a way that asymptotic optimality is retained 


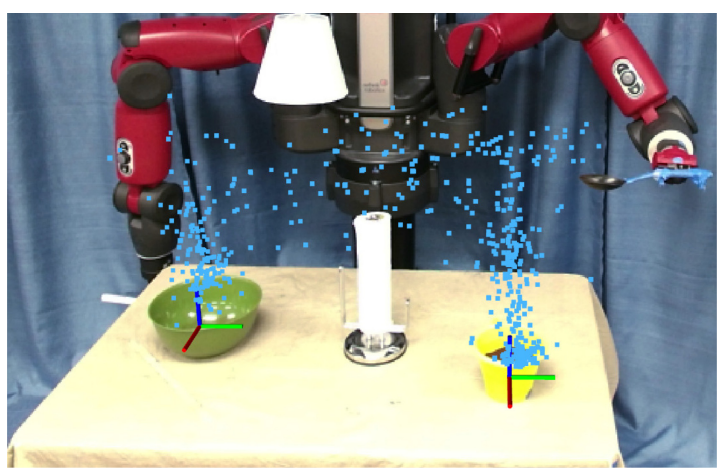

Fig. 5. End-effector positions of sampled configurations (shown in blue) from the biased distribution for the task of scooping powder from the yellow bucket into the green bowl. The distribution is dependent on the landmark positions. Sampling configurations in useful places enables us to build higher-quality roadmaps with fewer samples, which facilitates faster motion planning.

while producing samples that produce lower-cost edges and are thus more likely to be useful. This takes the place of the guiding path considered in [44].

The intuition behind the distribution that we construct is that, when lifted into motion feature space, it should approximate the distribution implied by the task model conditional on the poses of landmarks. Formally, we wish to sample a sequence $\left\{\mathbf{q}_{0}, \ldots, \mathbf{q}_{n}\right\}$ such that $f_{\mathbf{a}}\left(\mathbf{q}_{i}\right) \sim \mathcal{N}\left(\mu_{t_{i}}, \Sigma_{t_{i}}\right)$, where $t_{i} \sim \mathcal{U}(0, T)$. Recall that $f_{\mathrm{a}}$ lifts a configuration into motion feature space given landmark poses a.

To approximate this distribution, we work backwards. First, we sample $t_{i} \sim \mathcal{U}(0, T)$, followed by $\mathbf{y}_{i} \sim \mathcal{N}\left(\mu_{t_{i}}, \Sigma_{t_{i}}\right)$, then solve the following nonlinear least squares problem using a LevenbergMarquardt method:

$$
\mathbf{q}_{i}=\arg \min _{\mathbf{q}}\left(f_{\mathbf{a}}(\mathbf{q})-\mathbf{y}_{i}\right)^{\mathrm{T}} \Sigma_{t_{i}}^{-1}\left(f_{\mathbf{a}}(\mathbf{q})-\mathbf{y}_{i}\right) .
$$

This yields a configuration that, when lifted into motion feature space, is close to $\mathrm{y}_{i}$ in feature space under the metric implied by $\Sigma_{t_{i}}$. An example of the resulting distribution for one of the experimental scenarios is shown in Figure 5. Note that the distribution appears to follow the path of a successful execution but with greater variance where such variance would likely preserve plan success. For nonsingular $f_{\mathrm{a}}$ and $\Sigma_{t}$, the support of this distribution is all of $Q$; thus, asymptotic optimality holds.

In practice, the initial iteration can be sampled uniformly at random and the least squares problem need not be solved to great precision. We found that even very few iterations of the LevenbergMarquardt method result in distributions that sufficiently approximate the desired one.

\subsection{Real-Time Execution}

During execution, we repeatedly replan using the latest sensed information about the environment in a closed-loop manner, updating the path being executed with high frequency. In Figure 6, we show a schematic of the computation during real-time execution. Replanning comprises three logical steps: sensing, sampling, and searching.

4.4.1 Sensing. We refer to sensing as the general process of acquiring information about the environment used by the planning procedure. In our physical experiments, sensing consists of processing the raw point cloud from an RGBD sensor to locate task-relevant objects and build a collision model of the environment. These computations are performed asynchronously, ensuring 


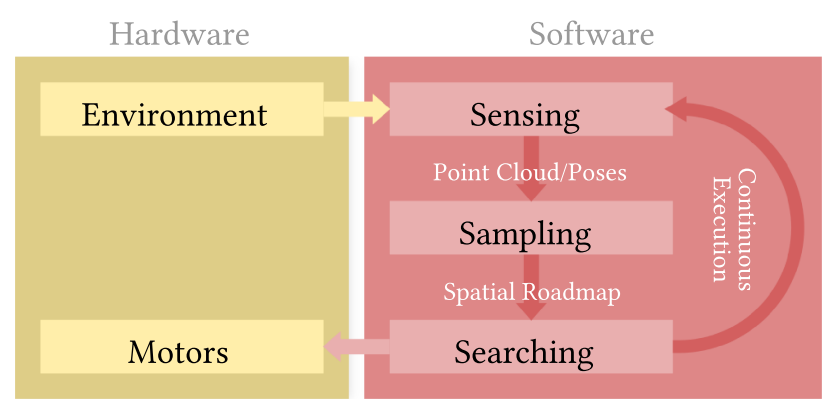

Fig. 6. Flow of computation during real-time execution.

that after each planning iteration, new environment information is available to immediately begin replanning.

4.4.2 Sampling. Sampling refers to the first step of building the spatial roadmap (as described in Section 4.1), which is randomly sampling configurations. Like prior work [6, 7], we use a fixed connection radius (sPRM) rather than a shrinking one ( $\left.\mathrm{PRM}^{*}\right)$ to retain asymptotic optimality [26] under the cost metrics that we consider (by metric equivalence). However, in contrast to prior work, our method builds a roadmap from scratch each cycle and does not reuse roadmaps from previous cycles. The rationale for this is twofold. First, in our problem, obstacles may move; thus, the validity of vertices and edges in the roadmap need to be reevaluated with each replanning step. This greatly reduces the benefit of retaining the previous roadmap. Second, the sampling distribution depends on the environment, yielding samples that are appropriate for a specific set of landmark poses. This not only means that the samples based on current landmark poses are more useful than those from prior work but that samples based on older landmark poses are less useful. Consequently, the benefit of building off the previous roadmap is outweighed by the cost incurred by the corresponding increase in roadmap size.

4.4.3 Searching. After the spatial roadmap is constructed, we perform a parallel bidirectional shortest-path search [16] on the spatiotemporal roadmap. Many calculations are performed lazily as edges of the graph are explored, including collision detection and edge cost evaluation. This is beneficial because fewer than $40 \%$ of the edges needed to be evaluated in order to identify the shortest path in our experiments, a positive consequence of the cost-space chasm induced by the task model.

Unlike many methods for real-time planning or control, our approach is globally optimal in that it selects the true minimal cost path present in the roadmap. Consequently, our method may consider multiple homotopic classes of paths and can avoid being trapped in a local minimum. Because we use a PRM, our approach is also asymptotically optimal in the sense that the plans produced approach optimality as the size of the roadmap used increases. While we only build relatively small roadmaps, asymptotic optimality of the planner allows the method to find better solutions, approaching the global optimum, as computational power increases. Our execution is real time in the sense that we impose a firm deadline on the planner, from sensing to execution, to ensure that the robot is never acting on sensor information that is excessively out of date. A missed deadline manifests as a pause while the robot waits for a new plan to be sent to the motors for actuation. If the replanning cycle finishes before the deadline, then the next replanning cycle begins immediately. In our implementation, we used a deadline of $250 \mathrm{~ms}$, although in our experiments we were able to achieve replanning cycles substantially faster than the deadline, on average. 
(b) Execution using our method.

(a) Example demonstrations.

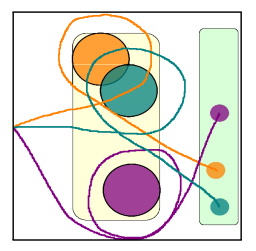

Environment 1 Environment 2 Environment 3

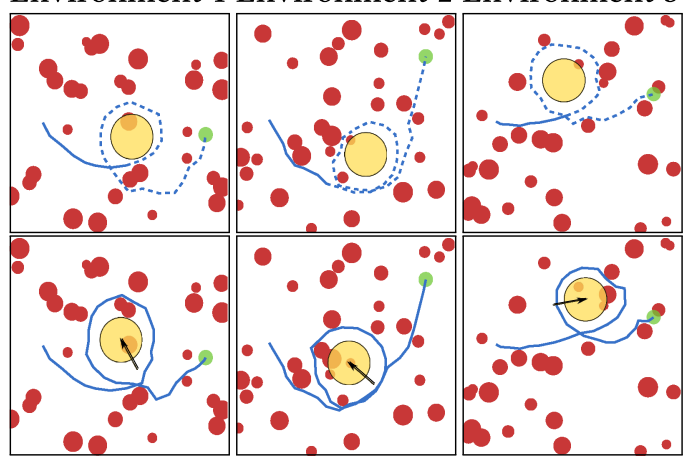

(c) Execution with only spatial Roadmap.

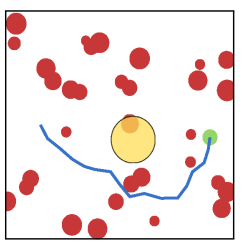

Fig. 7. (a) Three of the 7 demonstrations for the simulated navigation task as well as the beacon and goal sampling regions. The demonstrated paths start at a fixed location, move counterclockwise around a specified beacon, and then move to a specified goal. (b) Three executed trajectories (solid blue) and planned trajectories (dashed blue) around obstacles (red) to a goal (green) as computed by our method, before (top) and after (bottom) the beacon (yellow) was moved. (c) Execution without the temporal roadmap and using a temporal alignment heuristic [44].

\section{RESULTS}

To demonstrate the applicability of our method, we considered a simulated 2D navigation task and two physical tasks using the Baxter robot [38]. Planning was performed using a C++ implementation on a $3.4 \mathrm{GHz}$ Intel Xeon E5-1680 processor.

\subsection{Simulated Navigation Task}

We first consider a point robot that is to navigate on a 2D plane by starting at a fixed location, moving counterclockwise completely around a beacon without intersecting it and then stopping at a specified goal. In the learning phase, the feature space consisted of the absolute position of the robot and its position relative to two landmarks, corresponding to the beacon and intended goal position specified by the annotations. For the demonstrations, we manually performed 7 successful trajectories with beacon locations randomly sampled from the yellow region and goal locations randomly sampled from the green region in Figure 7(a).

In the execution phase, the test environment included 32 circular obstacles that were not present in the demonstrations. We randomly generated 15 test cases with different obstacle locations and with randomly chosen beacon and goal locations (sampled independently from the locations chosen for demonstrations but using the same regions). A quarter of the way through the trajectory (approximately when the robot begins encircling the beacon) we moved the beacon $10 \%$ of the width of the environment in a random direction.

The method successfully accomplished the task in all 15 test cases. Several representative executions are shown in Figure 7(b). Due to the density of the obstacles, in most of the test cases it was necessary for the method to change homotopic classes when replanning, illustrating the need for global motion planning rather than local refinement of plans.

This task also illustrates the need to incorporate task progress into the planning state. Planning in configuration space alone is not sufficient for correct execution because a successful path crosses itself by design. Using the temporal alignment heuristic from [44] skips an important portion of the task as shown in Figure 7(c). 

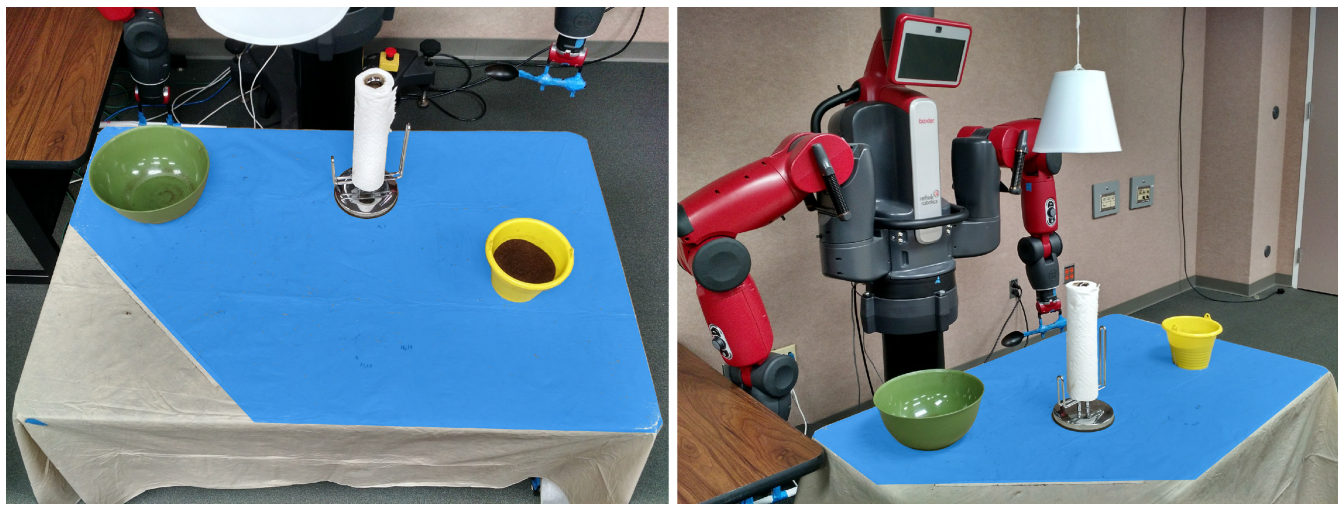

Fig. 8. Environment for the powder transfer task with the permissible region for both the green bowl and paper towel roll highlighted in blue. The permissible region is based on the reachable workspace of the Baxter's arm.
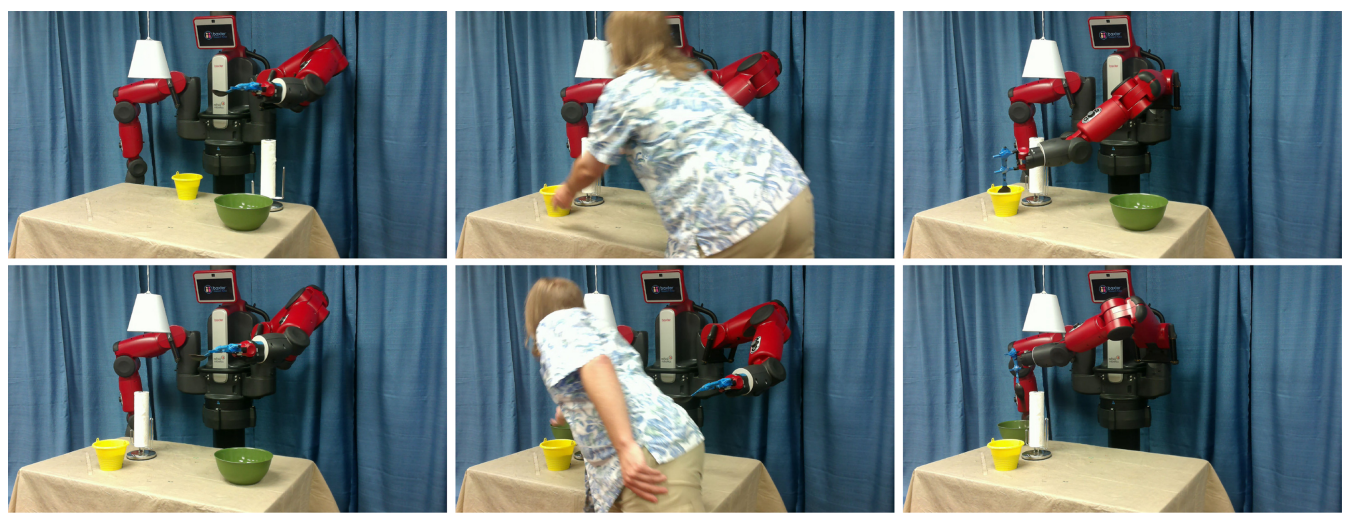

Fig. 9. Example execution for the powder-transfer task, from a bucket (yellow) to a bowl (green).

\subsection{Physical Powder-Transfer Task}

In the second task, the goal was to scoop powder from a small bucket and transfer it into a bowl with a spoon using the Baxter robot. To successfully perform the task, the robot was required to transfer the powder without spilling while avoiding obstacles in the environment. The feature space consisted of the position and orientation of the spoon relative to the bucket and bowl, for a total feature dimension of 12 . We provided the method with 13 successful kinesthetic demonstrations from which to learn.

At the beginning of execution, the green bowl was randomly placed on the reachable surface of the table (see Figure 8) and tracked using a Kinect sensor. For obstacles, we affixed a hanging lampshade above the table and randomly placed a vertical roll of paper towels. During task execution, when the robot positioned the spoon above the bowl but before it began dumping the contents, we quickly moved the bowl to a different location randomly sampled uniformly from the reachable surface of the table (see Figure 9).

We consider two variants of this task, as discussed below. 


\begin{tabular}{r|c|c|c} 
& $\begin{array}{c}\text { Success } \\
\text { Rate }\end{array}$ & $\begin{array}{c}\text { Avg. } \\
\text { Pauses }\end{array}$ & $\begin{array}{c}\text { Avg. } \\
\text { Update }\end{array}$ \\
\hline Full Method & $100 \%$ & 0 & $133 \mathrm{~ms}$ \\
No Bidirectional Search & $50 \%$ & 23 & $170 \mathrm{~ms}$ \\
No Obstacle Avoidance & $10 \%$ & 0 & $44 \mathrm{~ms}$ \\
No Biased Sampling & $0 \%$ & - & - \\
No Replanning & $0 \%$ & - & -
\end{tabular}

Fig. 10. Empirical results for the physical powder-transfer task with moving bowl and stationary yellow bucket and obstacle during execution, including success rates, average number of pauses, and average update periods for variants of the method. The additional failures without bidirectional search were due to insufficient reaction time when the bucket or bowl was moved. Without biased sampling, the method was unable to find successful plans even after 5 minutes of roadmap computation time. Similarly, replanning was required for success in every scenario owing to the motion of the bowl.

\begin{tabular}{r|c|c|c} 
& $\begin{array}{c}\text { Success } \\
\text { Rate }\end{array}$ & $\begin{array}{c}\text { Avg. } \\
\text { Pauses }\end{array}$ & $\begin{array}{c}\text { Avg. } \\
\text { Update }\end{array}$ \\
\hline Full Method & $100 \%$ & 0 & $111 \mathrm{~ms}$ \\
No Bidirectional Search & $30 \%$ & 27 & $148 \mathrm{~ms}$ \\
No Obstacle Avoidance & $10 \%$ & 0 & $45 \mathrm{~ms}$
\end{tabular}

Fig. 11. Empirical results for the physical powder-transfer task when moving the bowl, bucket, and obstacle during execution, including success rates, average number of pauses, and average update periods for variants of the method.

5.2.1 Moving Bowl During Execution. We first evaluated the method on the same set of 10 scenarios used in [6] for comparison. In these scenarios, the bowl was moved during execution but the yellow bucket was always placed in a single fixed location and the obstacles were stationary during execution.

We show empirical results for different variants of the method in Figure 10. For each method variant, we report the success rate, average update period (defined as the total time required to perform sensing and replan), and average number of pauses owing to missing a deadline (i.e., planning took longer than our deadline of $250 \mathrm{~ms}$ ). In some scenarios, the proximity of the paper towel roll and lampshade created a narrow passage in configuration space through which the robot arm needed to navigate to accomplish the task. Collision detection against a point cloud was the primary bottleneck, as reflected by the latency reduction when obstacle avoidance was disabled. For this reason, bidirectional search was very profitable because of the decrease in edges for which collision checking was required.

5.2.2 Moving Bowl, Bucket, and Obstacle During Execution. We next generalized the task by moving the bucket (before the powder was scooped), the roll of paper towels (at the same time as the bowl), and the bowl (as in the prior scenario) to random locations during execution. We also randomized the initial placement of the yellow bucket in these 10 scenarios. This task is shown in the video associated with this article.

Empirical results for these scenarios are shown in Figure 11. We first note that the average latencies were shorter than their counterparts in the stationary scenarios. This is because moving the objects often required the planner to spend more time in later parts of the task, where the number of remaining timesteps was fewer and thus the portion of the graph which needed to be searched was smaller. We note that this effect did not appear when obstacle avoidance was disabled, 

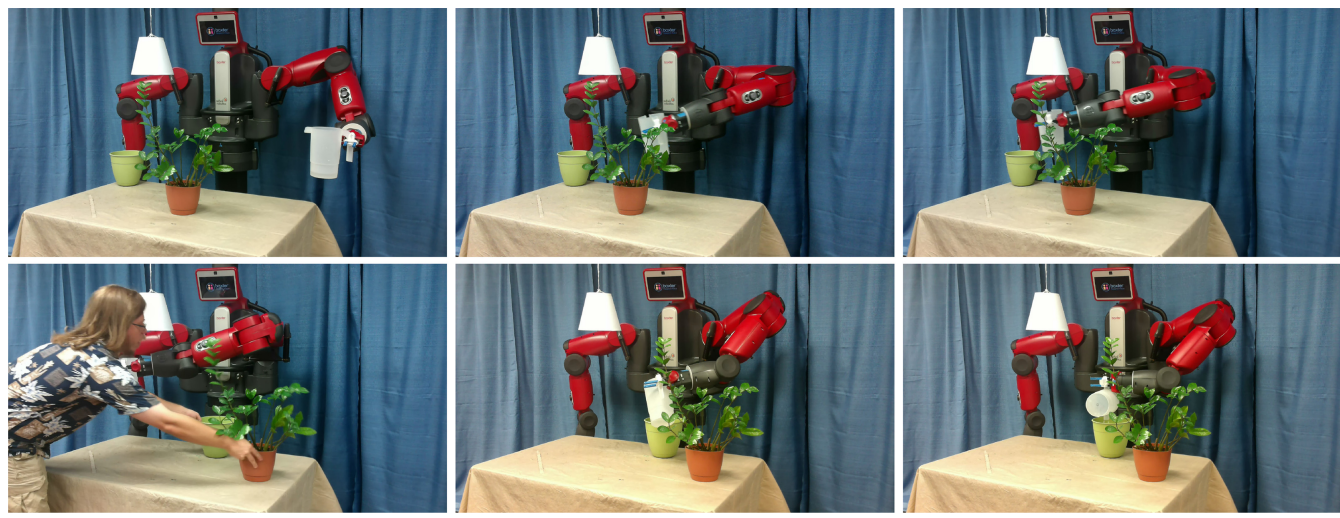

Fig. 12. Example execution for the liquid pouring task, from a pitcher into a pot (green).

\begin{tabular}{r|c|c|c} 
& $\begin{array}{c}\text { Success } \\
\text { Rate }\end{array}$ & $\begin{array}{c}\text { Avg. } \\
\text { Pauses }\end{array}$ & $\begin{array}{c}\text { Avg. } \\
\text { Update }\end{array}$ \\
\hline Full Method & $100 \%$ & 1 & $138 \mathrm{~ms}$ \\
No Bidirectional Search & $90 \%$ & 26 & $212 \mathrm{~ms}$ \\
No Obstacle Avoidance & $40 \%$ & 0 & $61 \mathrm{~ms}$
\end{tabular}

Fig. 13. Empirical results for liquid pouring task, including success rates, average number of pauses, and average update periods for variants of the method. The additional failure without bidirectional search was due to a failure to find a difficult narrow passage and likely only a result of the randomized nature of method.

likely because traversing edges occupies a smaller fraction of the computation time. However, there was also more variance in the latencies, as reflected in the increase in missed deadlines when bidirectional search was disabled. The success rate without bidirectional search was also lower than for the stationary scenarios because the robot failed to react in time to the motion of the bucket to satisfy the relatively narrow constraints required to successfully scoop powder from it.

\subsection{Physical Liquid Pouring Task}

We next evaluated the method on a liquid pouring task in which the Baxter robot pours liquid from a grasped pitcher into a pot without spilling. Here, the feature space consisted of the position and orientation of the pitcher relative to the bowl. We performed 11 successful demonstrations from which the task was learned. For the execution environment, we introduced as an obstacle a potted plant, which has complex geometry not amenable to modeling via geometric primitives but that can be sensed using Kinect (see Figure 12). As before, in each of the 10 scenarios, the pot and an obstacle were initially placed uniformly at random on the reachable surface of the table and then moved to different random locations, necessitating replanning. This task is shown in the video associated with this article.

Results for this task are shown in Figure 13. The size of the pitcher and plant obstacle produced multiple challenging scenarios (see the accompanying video) which required denser roadmaps to navigate. This, in turn, resulted in marginally longer planning times and a few missed deadlines. Without bidirectional search, planning was substantially slower but the success rate was comparable because this task was less time sensitive than the powder-transfer task. Obstacle avoidance was again crucial to successful execution in $60 \%$ of the scenarios. 


\section{CONCLUSION}

We presented a closed-loop motion planning approach that is applicable to execution of learned tasks in which the environment changes during execution. Using our method, the Baxter robot was able to successfully perform several learned tasks while avoiding moving obstacles and reacting to the motion of task-relevant objects whose locations were relevant to task success.

In future work, we plan to consider the problem of temporally extrapolating the state of the environment and incorporating the noise in these predictions to produce better plans and accommodate occlusion. We will also investigate methods to make replanning faster, including using bidirectional $A^{*}$ with a task-specific admissible heuristic. The additional performance could enable us to effectively react to faster moving objects while still succeeding in performing the learned task. We also plan to consider generalizations of the learned task model to attempt more complex tasks that require integration of task and motion planning.

\section{ACKNOWLEDGMENTS}

The authors thank Armaan Sethi for his assistance with the physical experiments and Jenny Womack, Alan Kuntz, Sachin Patil, Pierre Berthet-Rayne, Wen Sun, and Christophe Cordero for their input.

\section{REFERENCES}

[1] Ron Alterovitz, Sachin Patil, and Anna Derbakova. 2011. Rapidly-exploring roadmaps: Weighing exploration vs. refinement in optimal motion planning. In Proceedings of the IEEE International Conference on Robotics and Automation (ICRA'11). 3706-3712. DOI : http://dx.doi.org/10.1109/ICRA.2011.5980286

[2] Scott Benson. 1995. Inductive learning of reactive action models. In Proceedings of the International Conference on Machine Learning (ICML'95). 47-54. DOI : http://dx.doi.org/10.1.1.47.9180

[3] Dmitry Berenson, Thierry Siméon, and Siddhartha S. Srinivasa. 2011. Addressing cost-space chasms in manipulation planning. In Proceedings of the IEEE International Conference on Robotics and Automation (ICRA'11). 4561-4568. DOI : http://dx.doi.org/10.1109/ICRA.2011.5979797

[4] Dmitry Berenson, Siddhartha S. Srinivasa, Dave Ferguson, and James J. Kuffner. 2009. Manipulation planning on constraint manifolds. In Proceedings of the IEEE International Conference on Robotics and Automation (ICRA'09). 625632. DOI : http://dx.doi.org/10.1109/ROBOT.2009.5152399

[5] Julien Bidot, Lars Karlsson, Fabien Lagriffoul, and Alessandro Saffiotti. 2017. Geometric backtracking for combined task and motion planning in robotic systems. Artificial Intelligence 247, 229-265. DOI:http://dx.doi.org/10.1016/j. artint.2015.03.005

[6] Chris Bowen and Ron Alterovitz. 2014. Closed-loop global motion planning for reactive execution of learned tasks. In Proceedings of the IEEE/RSF International Conference on Intelligent Robots and Systems (IROS'14). 1754-1760. DOI : http://dx.doi.org/10.1109/IROS.2014.6942792

[7] Chris Bowen, Gu Ye, and Ron Alterovitz. 2015. Asymptotically-optimal motion planning for learned tasks using timedependent cost maps. IEEE Transactions on Automation Science and Engineering 12, 1, 171-182. DOI : http://dx.doi.org/ 10.1109/TASE.2014.2342718

[8] Felix Burget, Maren Bennewitz, and Wolfram Burgard. 2016. BI ${ }^{2} \mathrm{RRT}^{*}$ : An efficient sampling-based path planning framework for task-constrained mobile manipulation. In Proceedings of the IEEE/RSf International Conference on Intelligent Robots and Systems (IROS'16). IEEE, 3714-3721. DOI : http://dx.doi.org/10.1109/IROS.2016.7759547

[9] Sylvain Calinon, Florent D’halluin, Darwin G. Caldwell, and Aude G. Billard. 2009. Handling of multiple constraints and motion alternatives in a robot programming by demonstration framework. In Proceedings of the IEEE-RAS International Conference on Humanoid Robots. 582-588. DOI : http://dx.doi.org/10.1109/ICHR.2009.5379592

[10] Howie Choset, Kevin M. Lynch, Seth A. Hutchinson, George A. Kantor, Wolfram Burgard, Lydia E. Kavraki, and Sebastian Thrun. 2005. Principles of Robot Motion: Theory, Algorithms, and Implementations. MIT Press, Cambridge, MA.

[11] Jonathan Claassens. 2010. An RRT-based path planner for use in trajectory imitation. In Proceedings of the IEEE International Conference on Robotics and Automation (ICRA'10). 3090-3095. DOI: http://dx.doi.org/10.1109/ROBOT. 2010.5509596 
[12] Jonathan Claassens and Yiannis Demiris. 2012. Exploiting affordance symmetries for task reproduction planning. In Proceedings of the IEEE-RAS International Conference on Humanoid Robots (Humanoids). IEEE, 653-659. DOI: http://dx.doi.org/10.1109/HUMANOIDS.2012.6651589

[13] Ioan A. Şucan and Sachin Chitta. 2012. Motion planning with constraints using configuration space approximations. In Proceedings of the IEEE/RSF International Conference on Intelligent Robots and Systems (IROS'12). 1904-1910. DOI : http://dx.doi.org/10.1109/IROS.2012.6386092

[14] Neil T. Dantam, Zachary K. Kingston, Swarat Chaudhuri, and Lydia E. Kavraki. 2016. Incremental task and motion planning: A constraint-based approach. In Robotics: Science and Systems (RSS'16). Robotics: Science and Systems Foundation. DOI : http://dx.doi.org/10.15607/RSS.2016.XII.002

[15] Richard I. A. Davis and Brian C. Lovell. 2004. Comparing and evaluating HMM ensemble training algorithms using train and test and condition number criteria. Formal Pattern Analysis \& Applications 6, 4, 327-335.

[16] Dennis de Champeaux. 1983. Bidirectional heuristic search again. fournal of the ACM 30, 1, 22-32.

[17] A. Dobson and Kostas E. Bekris. 2013. A study on the finite-time near-optimality properties of sampling-based motion planners. In Proceedings of the IEEE/RSf International Conference on Intelligent Robots and Systems (IROS'13). 12361241. DOI : http://dx.doi.org/10.1109/IROS.2013.6696508

[18] Staffan Ekvall and Danica Kragic. 2008. Robot learning from demonstration: A task-level planning approach. International fournal of Advanced Robotic Systems 5, 3, 33. DOI : http://dx.doi.org/10.5772/5611

[19] Dave Ferguson, Nidhi Kalra, and Anthony Stentz. 2006. Replanning with RRTs. In Proceedings of the IEEE International Conference on Robotics and Automation (ICRA'06). IEEE, 1243-1248. DOI : http://dx.doi.org/10.1109/ROBOT.2006. 1641879

[20] Perttu Hämäläinen, Joose Rajamäki, and C. Karen Liu. 2015. Online control of simulated humanoids using particle belief propagation. ACM Transactions on Graphics 34, 4, 81 .

[21] Kris Hauser. 2012. On responsiveness, safety, and completeness in real-time motion planning. Autonomous Robots 32 , 1, 35-48. DOI : http://dx.doi.org/10.1007/s10514-011-9254-z

[22] Micha Hersch, Florent Guenter, Sylvain Calinon, and Aude Billard. 2008. Dynamical system modulation for robot learning via kinesthetic demonstrations. IEEE Transactions on Robotics 24, 6, 1463-1467. DOI:http://dx.doi.org/10 1109/TRO.2008.2006703

[23] Leonard Jaillet, Juan Cortes, and Thierry Simeon. 2010. Sampling-based path planning on configuration-space costmaps. IEEE Transactions on Robotics 26, 4, 635-646. DOI : http://dx.doi.org/10.1109/TRO.2010.2049527

[24] Leonard Jaillet and Josep Porta. 2012. Asymptotically-optimal path planning on manifolds. In Proceedings of Robotics: Science and Systems (RSS'12). Robotics: Science and Systems Foundation, Sydney, Australia. DOI : http://dx.doi.org/10. 15607/RSS.2012.VIII.019

[25] Rainer Jakel, Sven R. Schmidt-Rohr, Martin Losch, and Rudiger Dillmann. 2010. Representation and constrained planning of manipulation strategies in the context of programming by demonstration. In Proceedings of the IEEE International Conference on Robotics and Automation (ICRA'10). 162-169. DOI : http://dx.doi.org/10.1109/ROBOT.2010.5509959

[26] Sertac Karaman and Emilio Frazzoli. 2011. Sampling-based algorithms for optimal motion planning. International Journal of Robotics Research 30, 7, 846-894. DOI : http://dx.doi.org/10.1177/0278364911406761

[27] L. E. Kavraki, P. Svestka, J.-C. Latombe, and M. Overmars. 1996. Probabilistic roadmaps for path planning in highdimensional configuration spaces. IEEE Transactions on Robotics and Automation 12, 4, 566-580. DOI: http://dx.doi org/10.1109/70.508439

[28] Seyed Mohammad Khansari-Zadeh and Aude Billard. 2012. A dynamical system approach to realtime obstacle avoidance. Autonomous Robots 32, 4, 433-454. DOI : http://dx.doi.org/10.1007/s10514-012-9287-y

[29] Beobkyoon Kim, Terry Taewoong Um, Chansu Suh, and Frank C. Park. 2016. Tangent bundle RRT: A randomized algorithm for constrained motion planning. Robotica 34, 1, 202-225. DOI : http://dx.doi.org/10.1017/S0263574714001234

[30] D. Kulic, W. Takano, and Y. Nakamura. 2008. Incremental learning, clustering and hierarchy formation of whole body motion patterns using adaptive hidden Markov chains. International fournal of Robotics Research 27, 7, 761-784. DOI : http://dx.doi.org/10.1177/0278364908091153

[31] Fabien Lagriffoul, Dimitar Dimitrov, Julien Bidot, Alessandro Saffiotti, and Lars Karlsson. 2014. Efficiently combining task and motion planning using geometric constraints. International fournal of Robotics Research 33, 14, 1726-1747. DOI : http://dx.doi.org/10.1177/0278364914545811

[32] Steven M. LaValle. 2006. Planning Algorithms. Cambridge University Press, Cambridge, UK.

[33] Ryan Luna, Ioan A. Şucan, Mark Moll, and Lydia E. Kavraki. 2013. Anytime solution optimization for sampling-based motion planning. In Proceedings of the IEEE International Conference on Robotics and Automation (ICRA'13). 5068-5074. DOI : http://dx.doi.org/10.1109/ICRA.2013.6631301

[34] J. Mainprice, E. A. Sisbot, L. Jaillet, J. Cortés, R. Alami, and T. Siméon. 2011. Planning human-aware motions using a sampling-based costmap planner. In Proceedings of the IEEE International Conference on Robotics and Automation (ICRA'11). 5012-5017. DOI : http://dx.doi.org/10.1109/ICRA.2011.5980048 
[35] James D. Marble and Kostas E. Bekris. 2013. Asymptotically near-optimal planning with probabilistic roadmap spanners. IEEE Transactions on Robotics 29, 2, 432-444. DOI : http://dx.doi.org/10.1109/TRO.2012.2234312

[36] Anahita Mohseni-Kabir, Charles Rich, Sonia Chernova, Candace L. Sidner, and Daniel Miller. 2015. Interactive hierarchical task learning from a single demonstration. In Proceedings of the ACM/IEEE International Conference on Human-Robot Interaction (HRI'15). ACM Press, New York, New York, USA, 205-212. DOI : http://dx.doi.org/10.1145/ 2696454.2696474

[37] P. Pastor, L. Righetti, M. Kalakrishnan, and S. Schaal. 2011. Online movement adaptation based on previous sensor experiences. In Proceedings of the IEEE/RSF International Conference on Intelligent Robots and Systems (IROS'11). 365371. DOI : http://dx.doi.org/10.1109/IROS.2011.6095059

[38] Rethink Robotics. 2013. Baxter Research Robot. Retrieved April 26, 2018 from www.rethinkrobotics.com/ baxter-research-robot.

[39] Jonathan Scholz and Mike Stilman. 2010. Combining motion planning and optimization for flexible robot manipulation. In Proceedings of the IEEE-RAS International Conference on Humanoid Robots. 80-85. DOI : http://dx.doi.org/10 1109/ICHR.2010.5686849

[40] Ashwini Shukla and Aude Billard. 2012. Coupled dynamical system based arm-hand grasping model for learning fast adaptation strategies. Robotics and Autonomous Systems 60, 3, 424-440. DOI : http://dx.doi.org/10.1016/j.robot.2011.07. 023

[41] Siddharth Srivastava, Eugene Fang, Lorenzo Riano, Rohan Chitnis, Stuart Russell, and Pieter Abbeel. 2014. Combined task and motion planning through an extensible planner-independent interface layer. In Proceedings of the IEEE International Conference on Robotics and Automation (ICRA). IEEE, 639-646. DOI : http://dx.doi.org/10.1109/ICRA.2014. 6906922

[42] Mike Stilman. 2010. Global manipulation planning in robot joint space with task constraints. IEEE Transactions on Robotics 26, 3, 576-584. DOI : http://dx.doi.org/10.1109/TRO.2010.2044949

[43] Yuandong Yang and Oliver Brock. 2009. Elastic roadmaps-Motion generation for autonomous mobile manipulation. Autonomous Robots 28, 1, 113.

[44] Gu Ye and Ron Alterovitz. 2011. Demonstration-guided motion planning. In Proceedings of the International Symposium on Robotics Research (ISRR'11), Springer Tracts in Advanced Robotics, Vol. 100. 291-307. DOI : http://dx.doi.org/ 10.1007/978-3-319-29363-9_17

[45] Matt Zucker, James Kuffner, and Michael Branicky. 2007. Multipartite RRTs for rapid replanning in dynamic environments. In Proceedings of the IEEE International Conference on Robotics and Automation (ICRA'07). 1603-1609. DOI : http://dx.doi.org/10.1109/ROBOT.2007.363553

[46] Matt Zucker, Nathan Ratliff, Anca D. Dragan, Mihail Pivtoraiko, Matthew Klingensmith, Christopher M. Dellin, J. Andrew Bagnell, and Siddhartha S. Srinivasa. 2013. CHOMP: Covariant Hamiltonian optimization for motion planning. International fournal of Robotics Research 32, 9-10, 1164-1193. DOI : http://dx.doi.org/10.1177/0278364913488805

Received October 2017; accepted February 2018 